\title{
L'indication géographique, un instrument de pérennisation des productions localisées en Amazonie? Le cas de la farine de manioc de Cruzeiro do Sul (Acre, Brésil)
}

Mauro Almeida - Ph.D. em Antropologia Social pela University of Cambridge, com pósdoutorado na Universidade de Stanford e na Universidade de Chicago. Professor da UNICAMP.

Manuela Carneiro da Cunha - Professora na Universidade de Chicago. Doutora em Antropologia, professora da USP no Departamento de Antropologia.

Ludivine Eloy - Geógrafa-Agrônoma, Pesquisadora do Centre National de la Recherche Scientifique/CNRS, França.

Laure Emperaire - Doutora em Botânica, Pesquisadora do Institut de Recherche pour le développement/IRD, França.

Esther Katz - Antropóloga, Pesquisadora do Institut de Recherche pour le développement/IRD, França.

Jane Simoni - Doutoranda do Programa de Doutorado em Desenvolvimento Sustentável da UnB. Roberta Rizzi - Antropóloga, professora na. Unicamp.

Juliana Santilli - Professora do Curso de Direito Ambiental do Instituto Internacional de Educação do Brasil, Doutoranda no Programa de Doutorado da PUC, Paraná.

Lúcia van Velthem - Doutora em Antropologia, pesquisadora do MPEG, do Ministério de Ciência e Tecnologia/MCT e com experiência sobre cultura e sociedades indígenas na Amazônia.

\begin{abstract}
La création de la Réserve Extractiviste du Haut Juruá en 1990 a suscité un programme de recherches associant sciences humaines et sciences biologiques avec, comme question de fond, la prise en charge par les populations locales de la gestion d'aires. Cette recherche a constitué le point d'ancrage d'un autre programme sur la gestion de la diversité agricole. Il s'agissait en particulier de comprendre en l'application d'instruments de valorisation de productions localisées pouvait interférer avec les normes, perceptions et connaissances locales sur ces mêmes produits et quel était leur impact sur la biodiversité.
\end{abstract}

\section{Keywords}

Biodiversité, populations traditionnelles, Cruzeiro do Sul, farine de mandioc, Amazônia.

\section{Resumo}

A criação da Reserva Extrativista do Alto Juruá em 1990 suscitou um programa de pesquisas associando ciências humanas e ciências biológicas. Como questão de fundo, a responsabilidade das populações locais com a gestão do território. Esta pesquisa constituiu o ponto de encontro com um outro programa sobre a gestão da diversidade agrícola. Tratava-se em particular de compreender a aplicação de instrumentos de valorização de produção localizada, e como interferiam com as normas, as percepções e os conhecimentos locais sobre esses mesmos produtos e saber qual era seu impacto sobre a biodiversidade.

\section{Palavras-chave}

Biodiversidade, populações tradicionais, Cruzeiro do Sul, farinha de mandioca, Amazônia. 
Mauro Almeida / Manuela Carneiro da Cunha / Ludivine Eloy / Laure Emperaire / Esther Katz / Jane Simoni, Roberta Rizzi / Juliana Santilli / Lúcia van Velthem

\section{LES INDICATIONS GÉOGRAPHIQUES AU BRÉSIL ET LA FARINHA DE CRUZEIRO DO SUL}

Le système indications géographiques commence à acquérir une certaine notoriété au Brésil tant de la part des consommateurs que des producteurs. Il relève de la loi sur la propriété industrielle (9279/1996) et est géré par l'Institut National de la Propriété Industrielle. Deux phases se dessinent dans la courte histoire de son application. La première est concrétisée par la mise en place de quatre indications de provenance, vins du Vale dos Vinhedos dans le Rio Grande do Sul, viande de la Pampa Gaucha dans le même état, cachaça de Paraty dans celui de Rio de Janeiro et café du Cerrado à Minas Gerais, soit quatre produits originaires du sud et centre sud du Brésil. Ceux-ci, de forte portée économique, reposent sur une base sociale relativement restreinte puisque les quatre associations demandeuses ne comptaient respectivement, lors de leur demande de protection du nom géographique auquel leur produit est associé, que 27, 55, 9 et 156 producteurs (Mascarenhas, 2008).

A partir de 2005, il revient au Ministère de l'Agriculture, Élevage et Approvisionnement (Mapa) la charge de définir et de mettre en place une politique de valorisation des productions agricoles et de protection de la propriété intellectuelle et donc, entre autres instruments, des indications géographiques. C'est une deuxième phase qui se dessine. Elle est marquée par l'intervention d'un autre acteur majeur de cette politique, le Sebrae, ou Service Brésilien d'Appui aux micro et petites Entreprises (Lages et al., 2005). Environ cent productions localisées issues de 17 états brésiliens sont identifiées comme de potentielles IG. La gamme est vaste, elle inclut surtout des produits agricoles classiques mais aussi des produits miniers. Une douzaine de produits amazoniens est identifiée, parmi lesquels un cacao dit de várzea, l'açai-do-Pará (fruits du palmier Euterpe oleracea) et la farine de manioc de Cruzeiro do Sul (farinha de Cruzeiro do Sul) - produite dans la vallée du haut Juruá.

Néanmoins, l'analyse ici proposée est issue d'une autre préoccupation. La création de la Réserve Extractiviste du Haut Juruá en 1990 a suscité un programme de recherches associant sciences humaines et sciences biologiques avec, comme question de fond, la prise en charge par les populations locales de la gestion d'aires protégées ${ }^{1}$. Cette recherche a constitué le point d'ancrage d'un autre programme ${ }^{2}$

1 Programme financé par la Fondation McArthur "Can traditional forest-dwellers self-manage conservation areas Extractive Reserve, Acre, Brazil”, resp. M. Carneiro da Cunha, USP, M. Almeida, Unicamp, et K. Brown, Unicamp.

2 Programme PACTA, Populations locales, Agrobiodiversité et Connaissances Traditionnelles Associées em Amazonie brésilienne, CNPq - Unicamp / IRD, nº 492693 / 2004-8 avec des financements IRD, CNPq, ANR-Biodivalloc et BRG. Autorisation n ${ }^{\circ} 139$ publiée au DOU 
sur la gestion de la diversité agricole et ses formes de protection et de valorisation dans deux contextes amazoniens, le Rio Negro en Amazonie centrale et le haut Juruá dans le sud-ouest amazonien. Il s'agissait en particulier de comprendre en quoi l'application d'instruments de valorisation de productions localisées pouvait interférer avec les normes, perceptions et connaissances locales sur ces mêmes produits et quel était leur impact sur la biodiversité ${ }^{3}$. La farine de manioc dite de Cruzeiro do Sul se retrouvait à la croisée de ces deux parcours, l'un institutionnel - autour de l'implantation du système IG au Brésil-, l'autre scientifique et devenait ainsi un objet de choix pour comprendre l'émergence d'une production localisée. La question est d'autant plus d'actualité que le prochain désenclavement de la région, avec l'asphaltage de la route nationale BR 364 et, à plus long terme, l'ouverture de l'axe Atlantique-Pacifique qui reliera directement Cruzeiro do Sul à Pucallpa au Pérou, risque d'ouvrir tout le sud-ouest amazonien aux flux des produits issus de l'agriculture industrielle du sud et du centre du Brésil.

Deux questions orientent cet exposé. En quoi la farine de manioc est-elle une production localisée ? Et, quelle est la viabilité d'une indication géographique sur ce produit? Enfin, nous concluons sur la nécessité d'une approche différenciée des IG au Brésil dans un contexte social et territorial en construction.

\section{LA FARINHA DE CRUZEIRO DO SUL, UNE PRODUCTION LOCALISÉE?}

La farinha est selon les dénominations occidentales une semoule torréfiée. D’origine amérindienne, elle est probablement aujourd'hui l'aliment le plus populaire dans le Nordeste et en Amazonie brésilienne. Le manioc (Manibot esculenta Crantz) est une plante d'origine néotropicale, qui se décline en d'innombrables variétés regroupées en maniocs doux (à faible teneur en dérivés cyanhydriques, ce qui permet sa consommation après une simple cuisson) et maniocs amers qui doivent subir un processus complexe de détoxication. Les dérivés issus de l'un ou de l'autre de ces groupes sont extrêmement variés et vont des tubercules cuits pour les maniocs doux et, pour les doux comme les amers, à des bières, galettes, condiments, farines, chacun avec des procédés de préparation divers et répondant à des logiques de consommation différentes. La

(04/04/2006) : "Tout usage des informations exposées à des fins de bioprospection ou de développement technologique doit être soumis à l'obtention d'un accord préalable informé et d'un contrat d'utilisation du patrimoine génétique et de répartition des bénéfces établi avec les populations concernées, ainsi qu'à l'autorisation du CGEN. »

${ }^{3}$ Les résultats ici presentés sont issus de travaux de terrain menés entre 2006 et 2008 dans le village de São Pedro, à une cinquantaine de kilomètres par la route de Cruzeiro do Sul. Les enquêtes et relevés réalisés auprès d'une vingtaine d'agriculteurs ont porté sur les espaces cultivés, la diversité agricole mise à contribution, les techniques et objets employés et l'alimentation. Ces travaux ont été complétés par des enquêtes menées sur le marché de Cruzeiro do Sul. 
Mauro Almeida / Manuela Carneiro da Cunha / Ludivine Eloy / Laure Emperaire / Esther Katz / Jane Simoni, Roberta Rizzi / Juliana Santilli / Lúcia van Velthem

farine n'est donc que l'une des formes sous lesquelles se consomme le manioc, et quoique les populations locales la préfèrent fraîche et - malgré le rude labeur qu'elle entraîne- n’hésitent pas à la préparer très souvent, elle a ceci de particulier que, torréfiée plus longuement, elle peut se conserver longtemps. Ainsi les Tupinambá du XVIème siècle, préparaient-ils de la farine dite « de guerre » qui, très sèche, pouvait être emportée et subvenir à l'approvisionnement d'expéditions guerrières (Hans Staden, 1557, Jean de Léry, 1558, Magalhães Gandavo, 1575). À son tour, la colonisation de l'Amazonie et, en particulier, l'exploitation des produits forestiers (hévéas, noix d'Amazonie, etc.) s'est appuyée sur cet aliment local de longue conservation.

La forte demande en main d'œuvre pour l'exploitation des hévéas en Amazonie, alliée à une période de sécheresse dans le Nordeste, a été à la fin du dix-neuvième siècle à l'origine de plusieurs vagues de migrations des Nordestins vers les forêts de l'Amazonie du sud-ouest (Almeida et al., 2002). Cette migration est relativement tardive par rapport à l'histoire de l'exploitation du caoutchouc amazonien. Directement enrôlés dans la saignée de ces arbres, placés sous la coupe serrée d'un patron seringalista ou de ses contremaittres, l'activité agricole était interdite aux seringueiros et obligation d'acheter leur alimentation auprès des patrons, leur était faite. La farine de manioc venait, après un voyage fluvial de plusieurs mois ou semaines, de la région de Bragantina, près de l'embouchure de l'Amazone. Avec le déclin de l'extractivisme, à partir des années 1920, les activités agricoles se sont développées autour de Cruzeiro do Sul et la région est devenue le principal centre exportateur de farine de manioc de l'état. La région produit en outre un sucre brut dit gramixó, aussi exporté. Ces deux produits reposent sur des savoirs et une technologie d'essence nordestine et, pour le manioc, sur un matériel biologique amazonien.

Aujourd'hui les seringueiros et les agriculteurs de la région du haut Juruá, de Cruzeiro do Sul à la frontière péruvienne, ont pour aliment de base une farine de manioc particulière, faite à partir de variétés douces à chair de couleur blanche ou jaune clair. Malgré une très légère fermentation, elle est considérée comme une farine sèche (farinha seca) par opposition aux farines rouies, plus fréquentes en Amazonie (farinha d'água ou farinha puba), souvent de couleur jaune intense de par les variétés employées (Desmoulière, 2001). Elle s'apparente en cela aux farines du Nordeste semi-aride souvent sèches et de couleur ivoire. Le goût des farines amazonienne et nordestine tranche également : un peu acide

\footnotetext{
4 Pour une description des différents registres de savoirs et de pratiques des seringueiros et agriculteurs, ainsi que des Kashinawá, Katukina et Ashaninka du haut Juruá, voir Carneiro da Cunha \& Almeida, 2002.
} 
et granuleuse pour l'amazonienne, au goût moins marqué et de texture plus fine pour la nordestine. Celle produite à Cruzeiro do Sul est de texture intermédiaire, à granulation plutôt fine, croquante et de couleur ivoire. Dans son histoire comme son apparence, elle est un produit hybride entre le Nordeste et l'Amazonie, tout en étant considérée comme un produit régional amazonien.

Plusieurs éléments lui confèrent sa typicité. Tout d'abord, elle est préparée à partir de variétés locales, probablement d'origine amérindienne ${ }^{5}$. Comme dans toute l'Amazonie du sud-ouest, il s'agit de variétés douces (Emperaire, 2004) et à chair plutôt claire. Le choix du matériel biologique employé répond avant tout à des critères d'adaptation locale, en particulier à une fertilité des sols qui va en se dégradant, à une résistance des tubercules à la pourriture et à des critères de productivité mais peu à des critères de composition (teneur en fécule, en fibres et coloration) ou organoleptiques. Aucune variété n'est attachée à la préparation d'une farine particulière. Les farines reposent en général sur la combinaison de deux à trois variétés. À São Pedro, les variations entre stocks de variétés d'un agriculteur à l'autre sont faibles. Les nouveautés issues de l'Embrapa (organisme national de recherche agricole) ou celles obtenues par le jeu des déplacements et des échanges entre agriculteurs sont rapidement incorporées dans le stock des variétés et testées. Les choix variétaux apparaissent donc comme un facteur secondaire dans la perception locale de la qualité de la farine. Il en est autrement de son mode de préparation, élément au centre des notions de patrimoine et de production localisée.

Les femmes accordent plus d'attention que les hommes à la qualité des tubercules. Pour ceux-ci, la qualité d'une farine relève avant tout des artefacts utilisés, qu'il s'agisse de leur fabrication ou de leur action sur ceux-ci. Cette divergence entre hommes et femmes reflète évidemment les postes de travail occupés par les uns ou les autres et les connaissances mises en jeu. La chaîne opératoire est la suivante : les tubercules sont arrachés et transportés dans une charrette tirée par un bœuf, ou dans des paniers, jusqu'à la casa de farinha. Ils sont épluchés et lavés dans cette 'maison de farine', un bâtiment isolé de l'habitation, en général ouvert sur les côtés et couvert d'un toit de palmes ou de zinc. Ces deux opérations mobilisent femmes et enfants, qui animent éventuellement leur travail par des chants. Les hommes dirigent la suite des opérations. Les tubercules sont râpés, aujourd'hui avec une râpe à moteur. La pâte obtenue est mise dans

\footnotetext{
5 Les populations amérindiennes de la région, des groupes Pano et Arawak, cultivent plusieurs variétés de manioc et consomment les tubercules surtout cuits, avec donc une préparation presque quotidienne (Pantoja et al., 2002, Coffaci de Lima, com. pers., Pimenta, com. pers.). Les relevés font état de 22 variétés chez les Kashinawa, 14 chez les Katukina et 38 chez les seringueiros, soit une diversité régionale estimée à quelques dizaines de variétés (Pantoja et al., 2002).
} 
Mauro Almeida / Manuela Carneiro da Cunha / Ludivine Eloy / Laure Emperaire / Esther Katz / Jane Simoni, Roberta Rizzi / Juliana Santilli / Lúcia van Velthem

la presse en bois à vis (ou à levier) et soumise à une forte pression durant une douzaine d'heures. C'est là qu'elle subit un début de fermentation. Une fois la pâte compactée, la presse est dévissée et les blocs de manioc râpés à nouveau puis tamisés, dernière étape avant la cuisson. Cette dernière a lieu en deux fois, sur deux fours indépendants, chacun constitué d'une grande platine rectangulaire d'environ un mètre sur deux montée sur un foyer alimenté en bois de feu. La partie tamisée est d'abord rapidement saisie (escaldada) sur la platine où le feu est plus vif, à nouveau tamisée, puis transférée sur le feu plus doux où elle sera progressivement torréfiée. Il y a de nombreuses variantes autour de ce schéma : ainsi dans la Réserve Extractiviste, ce coup de chaud se fait sur un seul four mais en deux étapes. Il arrive que des producteurs négligent l'épluchage des tubercules ou le tamisage de la pâte, mais quoiqu'il en soit l'étape perçue comme décisive de la qualité d'une farine est sa cuisson. Certains producteurs optent pour saisir et torréfier une grande quantité à la fois. Le travail est plus rapide, mais la farine tend à conserver de l'humidité, ce qui se reflète dans la couleur, au toucher et dans sa durée de stockage. Après refroidissement dans une caisse en bois, elle est conditionnée en sacs. Les pratiques varient aussi selon les sous-produits désirés et privilégient un certain type de production. Ainsi l'extraction d'amidon pour la confection de biscuits, galettes ou bouillies pour les jeunes enfants est apprécié des femmes alors les hommes considèrent que l'incorporation de cet amidon dans la farine améliore sa texture et sa saveur (Pantoja et al., 2002). Toutefois, la production de farine n'est pas une seule suite d'opérations techniques menées par des individus. Elle met en jeu des relations entre individus et objets et entre objets eux-mêmes (Velthem, 2009).

L'effectivité de chaque objet dépend principalement de qui l'a confectionné et de son savoir-faire. Qui en est propriétaire ou qui l'utilise est d'importance moindre. Plus complexe est l'interactivité des objets entre eux. Ils forment des ensembles qui sont soumis, en raison des tâches compliquées qu'ils réalisent, à une discipline symbolique et effective et établissent ainsi des relations de partenariat et de complémentarité. Ces ensembles d'objets sont perçus par les producteurs du Juruá comme des familles qui 'vivent' sous un même toit, celui de la casa de farinha. Les trois principales sont celles du banc, ou établi, où les tubercules sont râpés et transformés en pâte, celle de la presse et celle du four. A l'instar des humains, les artefacts 'travaillent' et sont liés par des relations de 'parenté' : ce n'est qu'en 'famille' qu'ils deviendront pleinement efficaces. Cette conception de l'organisation des objets se calque sur le fonctionnement du système de production local qui repose sur l'unité domestique. La production de farine réunit le groupe familial avec les parents, enfants, conjoints et petits- 
enfants et, comme l'ont signalé Pantoja et al. (2002) et Rizzi (2006), permet la transmission intergénérationnelle des savoirs.

Dans toutes les familles, le cabeça est le géniteur, ou la génitrice, de celle-ci. Il est représenté par l'objet qui exécute la fonction à laquelle se destine l'ensemble de la famille d'instruments. Il s'agira du rouleau denté qui râpe le manioc (le caitetu), de la presse ou de la platine. Sont frères ou sœurs au sein d'une même famille les objets de même nom, par exemple tous les tamis. Les fours sont frères eux aussi : le four benjamin est le plus petit, celui réservé à la saisie de la farine. L'organisation spatiale de la casa de farinha affecte humains comme objets. La partie froide abrite toutes les étapes jusqu'au dernier tamisage et celle chaude, assoviée aux fours, inclut l'enlèvement des braises et cendres une fois la farine mise en sac. Les activités féminines et masculines se déroulent ainsi dans des espaces particuliers. Humains comme objets ne peuvent transiter entre les deux sphères antagoniques du froid et du chaud et ils doivent éviter d'être sujets à leur double action. Cette séparation a pour conséquence une spécialisation des tâches entre les prenseiros, les presseurs, et les forneiros, les 'enfourneurs'.

L'univers matériel de la farine de manioc est donc extrêmement structuré tant du point de vue conceptuel que relationnel. Les objets sont des vecteurs actifs de la valeur de la farine. Eux-mêmes valorisés, ils transfèrent cet attribut au produit préparé. Les instruments sont perçus par les agriculteurs comme les éléments qui organisent la chaîne opératoire qui va du manioc à la farine et agissent sur elle. Comme l'un d'entre eux l'affirme 'c'est la platine du four qui décidera si la farine est bonne'. Les objets ne sont pas passifs, ils agissent et ne se laissent que difficilement dominer. Peut-être est-ce là une métaphore de la difficulté à produire une farine de qualité.

La farine de manioc est la base de l'alimentation des seringueiros et agriculteurs d'origine nordestine qui la produisent pour leur consommation. C'est aussi pour eux une façon de se démarquer des Indiens de la région qui, eux, mangent les tubercules cuits.

Les agriculteurs péri-urbains destinent au commerce une farine fine et colorée au curcuma, mais portent leur goût sur une farine plus granuleuse et blanc-ivoire. La farine de manioc est considérée comme un aliment 'faible' car elle ne peut être consommée toute seule, mais elle demeure indispensable à tout repas. Plus en amont, dans la Réserve Extractiviste, elle constitue l'apport calorique principal (Araújo et al., 2002). Autour de la ville, lorsque les ressources, financières ou agricoles, de la maisonnée le permettent, la farine est saupoudrée sur le riz et les haricots comme dans le Nordeste. Elle est souvent présentée dans la farinheira, petite boîte circulaire et aplatie à couvercle, aujourd'hui souvent 
Mauro Almeida / Manuela Carneiro da Cunha / Ludivine Eloy / Laure Emperaire / Esther Katz / Jane Simoni, Roberta Rizzi / Juliana Santilli / Lúcia van Velthem

en aluminium, modèle que l'on retrouve aussi dans le Nordeste. Les féculents sont consommés de préférence avec de la viande de gibier, parfois du poisson. Du Nordeste, les habitants ont également importé diverses recettes à base de maïs et les techniques de transformation de la canne à sucre. Le sucre mascave est une douceur qui se prend avec un peu de farine. L'alimentation repose sur un canevas nordestin auquel ont été incorporées des ressources amazoniennes, poisson, gibier, fruits (uvaia, сирuaçu, bacuri,...) et fruits de palmier (bacaba, pataua, açấ) appréciés eux aussi avec de la farine.

Ainsi cette farine, dont la renommée dépasse le cadre local, a pour origine une superposition de variétés, savoirs et techniques de différents registres. Elle manifeste un lien entre les descendants de Nordestins et un nouveau territoire et met en valeur des savoirs qui leurs sont propres. C'est au sein de sa préparation que se forge l'identité de ce produit régional, et qu'il devient la farinha de Cruzeiro do Sul.

\section{LES POUVOIRS PUBLICS ET LA FARINE}

Les six communes (municípios) qui composent la région du haut Juruá produisent $187400 \mathrm{t}$ (IBGE, 2007), soit moins d'un tiers de la production de l'état d'Acre qui elle-même, avec 614000 t ne constitue que 2,5\% de la production brésilienne. Du point de vue national, il s'agit d'un produit d'importance limitée mais sur le plan local, cette farine représente 70 à $80 \%$ du revenu des agriculteurs, mobilise l'essentiel de la main d'œuvre et c'est sur elle que repose la sécurité alimentaire des populations locales. Elle est aujourd'hui commercialisée par voie terrestre à Rio Branco, capitale de l'Acre, Porto Velho, capitale du Rondônia et, par voie fluviale, à Manaus, capitale de l'Amazonas. C'est sur un commerce actif mais mal rémunéré que se greffent les différentes initiatives de valorisation du produit. Celles-ci sont cependant anciennes puisque Teixeira Guerra (1955) fait état en 1952 de distribution de matériel pour l'agriculture et la transformation du manioc.

L'histoire récente de la farinha est marquée par plusieurs tentatives de valorisation synthétisées à partir des données de Simoni (2009) et Rizzi (2006). En 1997 est créée la Casavaj ou Coopérative des Agriculteurs et Seringueiros de la Vallée du Juruá avec pour objectif d'organiser sa commercialisation par une vente directe aux distributeurs. Pour cela, elle a reçu à partir de 1999 d'importants appuis sous le gouvernement de Jorge Viana du Parti des Travailleurs, dit le 'Gouvernement de la Forêt'. A partir de 2004, l'Anac, Agência de Negócios do Governo do Acre, chargée de la promotion des produits de l'Acre, se mobilise autour de cette farine. Son objectif est de former les agriculteurs à de « bonnes 
pratiques » pour la production et l'hygiène. Elles se concrétisent par l'installation de nouvelles casas de farinha, une aide au transport du produit et l'accès au crédit. Une autre ligne d'action vise à promouvoir la farinha par la création d'emballages et à identifier les de potentiels acheteurs. Une unité de conditionnement est installée à Cruzeiro do Sul, la farine y est empaquetée en sacs de $1 \mathrm{~kg}$ ou en boîtes de métal du type farinheira de $250 \mathrm{~g}$ à destination des grandes villes brésiliennes. Une brève articulation se dessine avec la chaîne de distribution Pão de Açúcar et sa ligne de produits Caras do Brasil (Visages du Brésil) (Página 20, 2004).

Malgré ces avancées qui, selon les agriculteurs, ont eu un effet positif sur le prix de la farine, une mauvaise gestion alliée à un manque d'expérience mène la coopérative à la faillite en 2005. Son origine, une initiative gouvernementale et non la mobilisation d'un groupe de producteurs locaux, explique aussi cet échec. La même année est créée une autre coopérative, la Cooperfarinha. En 2007, celle-ci était néanmoins dans une passe difficile avec 27 associés au lieu des 55 du moment de sa fondation. L'insolvabilité de certains de ses associés, anciens membres de la Casavaj, limite l'accès au crédit et la constitution d'un fonds de roulement et la fragilise. Dans ce cadre, nombre des associés reviennent à l'ancien système et vendent leur farine à des intermédiaires ou marreteiros, et ne commercialisent qu'une fraction réduite de leur production via la coopérative. Ils subissent alors la forte volatilité des prix d'achat de la farine (saisonnière et interannuelle), due, entre autres facteurs, aux spéculations des commerçants qui dictent leur prix en fonction des possibilités d'écoulement du produit à Rio Branco et Porto Velho, écoulement à son tour conditionné par l'état de la BR 364 (Oliveira et al., 2004).

Le défi que tente de relever cette nouvelle structure est de commercialiser une farine de qualité au meilleur prix. Néanmoins pour les responsables, l'idée de farine de qualité repose avant tout sur la notion d'homogénéité, de respect de normes sanitaires et de visibilité du produit. Pour répondre à ces critères, les agriculteurs doivent suivre une formation sur les 'bonnes pratiques de production', dispensée par l'Embrapa, le Sebrae et le Mapa et transformer leur manioc dans des casas de farinha modernisées ${ }^{6}$. Leur effort n'est cependant pas reconnu par une meilleure rémunération. Le Sebrae intervient aussi, à travers le Projeto Farinha, pour commercialiser le produit dans les villes de l'Acre. Il met à la disposition des agriculteurs des sacs de $1 \mathrm{~kg}$ estampillés Farinha do Acre et non Farinha de Cruzeiro do Sul, sans prendre en compte le caractère localisé de cette production. C'est dans ce contexte qu'intervient la quatrième étape de la

\footnotetext{
${ }^{6}$ Toutefois, selon V. Alvares de l'Embrapa (com. pers.), les farines produites dans les deux situations, traditionnelle ou modernisée, sont de bonne qualité sanitaire.
} 
Mauro Almeida / Manuela Carneiro da Cunha / Ludivine Eloy / Laure Emperaire / Esther Katz / Jane Simoni, Roberta Rizzi / Juliana Santilli / Lúcia van Velthem

valorisation de ce produit, celle de sa possible reconnaissance comme Indication Géographique qui commence en 2008.

\section{LA FARINHA ET LA FORÊTT}

La production de farinha, victime de son succès commercial et de la non prise en compte par les institutions intervenantes des conditions écologiques de sa production, se trouve aujourd'hui dans une situation de blocage. Là où cela était possible, il y a eu extension des surfaces cultivées prises sur la forêt selon une logique de front pionnier. Compte tenu de cette dynamique, on peut s'interroger sur la durabilité environnementale, sociale et économique de la production de farinha.

Les temps de jachère sont relativement courts, de l'ordre de deux à cinq ans, d'où une réduction de la fertilité des sols après quelques cycles de culture, une augmentation des adventices, et donc de la charge de travail. Les anciens abattis sont en grande partie envahis par le capim de burro (Andropogon sp.) et la pluma (Pteridium aquilinum (L.) Kuhn.), très agressive. De la forêt, il ne reste guère que les étroites bandes de forêt-galerie aujourd'hui protégées. Quelques agriculteurs pratiquent un labourage mécanisé mais sont rapidement confrontés à des problèmes de compactation du sol et d'envahissement accéléré de la pluma. Dans les zones anciennement exploitées, la production de farine a fortement diminué, car elle n'est plus tellement guère rentable. Le sac de $50 \mathrm{~kg}$ d'une farine de bonne qualité est vendu environ $35 \mathrm{R} \$$ ce qui ne compense pas le travail investi (Maciel et al., 2007), situation pleinement reconnue par la plupart des agriculteurs avec qui des entrevues ont été menées.

Cette situation est compensée par l'ouverture de nouveaux abattis dans les forêts plus lointaines des bourgs mais aboutit à la même situation de blocage après une quinzaine d'années ou vingtaine d'années de mise en valeur (selon la taille du lot). En effet, même si les exploitations situées dans les colonies agricoles récentes disposent encore des réserves importantes de forêt et des recrûs de type forestier, il apparaît qu'en moins de dix ans, une grande partie des terres a déjà été défriché au moins une fois, ce qui corrobore les données de Brondizio et al. (2002) sur la dynamique des fronts pionniers. Les temps de culture, de 1 à 3 ans, alternent avec des jachères de 1 à 4 ans. Le manioc devient la culture exclusive après 3 à 4 cycles, et laisse place aux pâturages après 5 à 6 cycles ( soit 15 à 20 ans après le premier défrichement). Si l'on considère qu'une famille défriche en moyenne 2 ha par an (pour 3 à 5 ha cultivés au total), et que les 2 premiers ha sont « immédiatement » convertis en pâturage, un lot de 15 ha de forêt devient inapte à la culture du manioc en 25 ans environ, soit l'échelle d'une génération. L'étude 
menée à São Pedro montre que ce sont en général des couples jeunes, avec très peu de capital qui s'installent dans les zones peu accessibles encore forestières (Eloy, 2009). Auparavant, ils dépendaient d'une très faible production de manioc dégagée sur les terrains, déjà épuisés, de leurs parents. Avec l'accès à ces nouvelles terres, ce sont souvent les deux générations qui déplacent leurs parcelles ou même leur résidence principale. L'essentiel du manioc aujourd'hui commercialisé provient de ces nouvelles zones où la disponibilité en terres permet d'avoir de grandes parcelles, de 3 à 5 ha, avec une productivité élevée (de 5 à 8 tonnes de farine par hectare contre de 2,5 à 3 dans les zones plus anciennes).

Aujourd'hui, la culture, la transformation et la commercialisation du manioc répond à la reproduction du modèle d'occupation d'un espace forestier. L'existence d'une réserve de terres forestière, alliée à des politiques publiques qui favorisent l'ouverture de voies d'accès, permettent la création régulière de nouveaux assentamentos (colonies agricoles). Cependant, on observe auprès des jeunes une tendance à la multi ou bi-résidence entre la zone rurale et la zone urbaine. Ceci indique peut-être une tendance à la diminution du rôle de la farine de manioc dans la constitution des revenus des ménages, et plus généralement, une moindre dépendance envers l'agriculture au sein de familles pluriactives, ce qui pourrait ralentir l'avancée du front pionnier comme dans d'autres régions d'Amazonie brésilienne (Arnaud de Sartre, 2006).

Sous l'impulsion des organismes de développement agricole et face à l'impossibilité de maintenir l'ancien modèle de production par manque de nouvelles terres, mais aussi en raison des limitations juridiques de déboisement, la réponse aujourd'hui apportée par les agriculteurs est de diversifier les productions et les formes de produire (pisciculture, engrais verts, début d'une mécanisation, développement de productions maraîchères). Cependant, il s'agit d'une période d'expérimentation et la capacité des engrais verts à assurer une production durable de manioc sur des parcelles envahies par les adventices doit encore être prouvée et acceptée par les agriculteurs (surcroît de travail, dépendance envers les tracteurs pour la préparation du sol, manque de semences, clôture des parcelles pour éloigner les bovins, faible maitrise des feux, ravageurs).

\section{CONCLUSION}

La brève description ici faite de la farinha de Cruzeiro do Sul souligne qu'elle répond aux critères de 'dénomination d'origine' tels que la législation les stipule (Santilli, 2005). Ce n'est pas seulement l'origine géographique du produit qui lui confère son caractère singulier, ce qui le ferait relever de 'l'indication de provenance', mais bien une typicité liée à des facteurs naturels et culturels, qui en 
Mauro Almeida / Manuela Carneiro da Cunha / Ludivine Eloy / Laure Emperaire / Esther Katz / Jane Simoni, Roberta Rizzi / Juliana Santilli / Lúcia van Velthem

font un produit différencié au sein des farinhas amazoniennes. D'autres farinhas pourraient revendiquer une telle reconnaissance, par exemple celle de Uarini, au grain sphérique, celle du Pará, celle du Rio Negro - avec ses variantes - ou celle dite do toco mole de la région de Maués. Comme pour nombre d'autres produits l'identité d'une farinha est reconnue à l'échelle domestique, de par les modes de faire, le soin apporté à la réalisation des différentes étapes ou une innovation personnelle et à l'échelle régionale par les caractéristiques générales des variétés employées, les conditions écologiques et un savoir faire partagé et reconnu comme tel.

La reconnaissance d'une indication géographique n'a pas encore été discutée avec les agriculteurs locaux mais, en l'absence d'autres opportunités de valorisation de leurs produits, il est fort probable qu'elle recevrait un accueil positif. Les plaintes contre l'usurpation du nom de Cruzeiro do Sul par des farines de moindre qualité produites dans la vallée de l'Acre ou dans l'état de Rondônia et l'épisode de l'emballage des farines locales sous le nom de farinha do Acre soulignent le lien entre territoire et produit. L'existence d'une IG répondrait au souci des agriculteurs de faire reconnaître leur savoir et de valoriser économiquement leur production. Pour les pouvoirs publics, l'attribution d'une indication géographique relève de la consolidation économique de filières productives (les arranjos produtivos locais) portées par une identité régionale. Mais pour l'instant c'est l'adéquation du produit à des normes sanitaires et visuelles qui a été privilégiée sans prendre en compte le fait que cette production de farinha s'inscrit dans un modèle particulier d'occupation de la terre et que sa durabilité dans les conditions actuelles, n'est pas assurée.

Les bases territoriales de cette indication géographique doivent encore être discutées. Deux dynamiques se superposent. L'une s'inscrit dans l'histoire de la farinha élaborée dans le haut Juruá et qui a abouti à différencier la farine produite dans la zone qui est aujourd'hui celle de la Réserve Extractiviste de celle des alentours de Cruzeiro do Sul. L'autre qui résulte des actions récentes des institutions qui privilégient un produit issu de systèmes de production plus intensifs en capital et en main d'oeuvre, et répondant à des normes d'hygiène et d'apparence, ce qui restreint les posssibilités d'accès à ce marché des producteurs les plus démunis. Les conditions sont réunies pour une 'spéciation' de cette farinha ce qui va à l'encontre du maintien d'une diversité biologique et culturelle régionale. Ce produit issu de savoirs 'traditionnels' deviendrait, et est en passe de devenir, un produit issu 'de savoirs techniques' avec un difficile dialogue entre ces deux registres. Le conflit potentiel entre l'augmentation d'échelle d'une production localisée vis-à-vis des considérations d'ordre biologique et 
culturel doit faire l'objet d'une discussion approfondie et peut être envisagée en complément d'autres voies de valorisation économique ou culturelle (agriculture biologique, enregistrement au patrimoine immatériel).

\section{BIBLIOGRAPHIE CITÉE}

ALMEIDA, M. SCHEIBE WOLFF, C., LOZANO COSTA, E., PANTOJA FRANCO, M., 2002, 'Habitantes: os seringueiros', In. Carneiro da Cunha M. \& M. Almeida, (eds.), Enciclopédia da Floresta, São Paulo, Companhia das Letras, pp.105-146.

ARAÚJO, G.J., MENDES, M.K., PANTOJA, M., COFFACI DE LIMA, E., CARNEIRO DA CUNHA, M., BARBOSA DE ARAUJO, M., SCHEIBE WOLFF, C., 2002, Cozinhar e comer. In. Carneiro da Cunha, M. \& Almeida, M., Enciclopédia da floresta., eds. São Paulo: Companhia das Letras, pp. 359-385.

ARNAUD de SARTRE, X., 2006, Fronts pionniers d'Amazonie, les dynamiques paysannes au Brésil, Paris, CNRS.

BRONDIZIO E., McCRACKEN, S.D., MORAN, E.F., NELSON, D.R., SIQUEIRA, A.D., RODRIGUEZ-PEDRAZA, C., 2002, The colonist footprint: Toward a conceptual framework of deforestation trajectories among small farmers in frontier Amazônia, In. Wood, C. \& R. Porro (eds.), Deforestation and land use in the Amazon, Gainsville, University Press of Florida, pp. 133161.

CARNEIRO DA CUNHA M. et ALMEIDA, M., (eds.), Enciclopédia da Floresta, o alto Juruá: práticas e conhecimentos das populações. São Paulo, Companhia das Letras, 735 p.

DESMOULIERE, S., 2001, Approche ethnobotanique de la diversité variétale du manioc en Amazonie centrale: gestion et perspectives de conservation. Thèse de doctorat en Ethnobiologie, Paris, Muséum National d'Histoire Naturelle, 360 p.

ELOY, L., 2009, Rapport de mission sur la mission réalisée en novembredécembre 2008, dans la région de Cuzeiro do Sul, Pacta (IRD-Unicamp) / Biodivalloc, Montpellier-Paris, 44 p. non publié

EMPERAIRE, L., 2004, Elements for a discussion on the conservation of agrobiodiversity: the exemple of manioc (Manihot esculenta Crantz) in the 
Mauro Almeida / Manuela Carneiro da Cunha / Ludivine Eloy / Laure Emperaire / Esther Katz / Jane Simoni, Roberta Rizzi / Juliana Santilli / Lúcia van Velthem

Brazilian Amazon. In. A. Veríssimo, A. Moreira, D. Sawyer, I. dos Santos, \& L. P. Pinto, eds. Biodiversity in the Brazilian Amazon - Assessment and priority actions for conservation, sustainable use and benefit sharing. São Paulo: Estação Liberdade, pp. 224-233.

IBGE, 2007, http:/ /www.ibge.br, consulté le 8 mai 2009

LAGES, V., L. LAGARES, \& BRAGA, C.L., 2005, Valorização de produtos com diferencial de qualidade e identidade: indicações geográficas e certificações para competitividade de negócios. Brasília: Sebrae, 227 p.

LERY, J. (de), 1992 (1580), Histoire d'un voyage fait en la terre du Brésil -1557, édité par F. Lestringant, Presses du Languedoc/Max Chaleil éditeur.

MACIEL, R.C., 2007, Diagnóstico Sócio-Econômico do Vale do Juruá períodos 1999/2000 e 2000/2001. Análise Econômica de Sistemas Básicos de Produção Familiar Rural no Estado do Acre - ASPF. ZEAS/PMRB. 2007.

MAGALHÃES GÂNDAVO, P. de, 1980 [1576] - Tratado da Terra do Brasil; História da Província Santa Cruz, Belo Horizonte, Ed. Itatiaia, 150 p.

MASCARENHAS, G., 2008, As indicações geográficas no Barsil, caracterização, desafios e oportunidades, communication au Séminaire International, Indicações Geográficas no Brasil: perspectivas e desafios, 6-7/ 11 / 2008, Florianópolis. Página 20, 2004, http://www.pagina20.com.br/15042004/c_0215042004.htm PANTOJA, M., AQUINO, T., ALMEIDA, M., 2002, 'Botar roçados', In. Carneiro da Cunha. M. \& M. Almeida (eds.), Enciclopédia da Floresta, São Paulo, Companhia das Letras, pp. 249-283.

RIZZI, R. 2006, A farinha de mandioca de Cruzeiro do Sul: um estudo de caso sobre mudanças culturais e ecológicas. Rapport Biodivalloc, IRD-Unicamp, Campinas-São Paulo, 14 p.

SANTILLI, J., 2005, As indicações geográficas e as territorialidades específicas das populações tradicionais, povos indígenas e quilombolas. In. Lages, V., L. Lagares, \& Braga, C.L., Valorização de produtos com diferencial de qualidade e identidade: indicações geográficas e certificações para competitividade de negócios. Brasília: Sebrae, pp. 189-203. 
SIMONI, J., 2009, A multidimensionalidade da valorização de produtos locais: implicações para políticas públicas, mercado, território e sustentabilidade na Amazônia, Thèse de doctorat, Centre de Développement Durable, Université de Brasília.

STADEN, H., 2008 [1557]. Duas viagens ao Brasil. Primeiros registros sobre o Brasil (trad. A. Bojadsen). Porto Alegre: L\&PM.

TEIXEIRA GUERRA, A., 1955, Estudo Geográfico do Territorio do Acre. Rio de Janeiro, Serviço Grafico do Instituto Brasileiro de Geografia e Estatistica, Biblioteca Geografica Brasileira, Publ. 11.

VELTHEM, L. H. van, 2008, Farinha, casas de farinha e objetos familiares em Cruzeiro do Sul - Acre. Revista de Antropologia São Paulo: USP, 50 (2): 605631. 